\title{
microRNA-342-3p targets FOXQ1 to suppress the aggressive phenotype of nasopharyngeal carcinoma cells
}

\author{
Zheqing Cui and Yulin Zhao*
}

\begin{abstract}
Background: microRNA (miR)-342-3p is frequently dysregulated in human cancers. In the present study, we aimed to explore the expression, prognostic significance, and biological relevance of miR-342-3p in nasopharyngeal carcinoma (NPC).

Methods: We examined miR-342-3p expression in 79 paired NPC specimens and corresponding normal tissues and analyzed its prognostic impact on overall survival of NPC patients. Gain- and loss-of-function experiments were conducted to determine the biological roles of miR-342-3p.

Results: Compared with matched normal nasopharyngeal tissues, miR-342-3p was significantly downregulated in NPC $(P=0.0038)$. Low miR-342-3p expression was significantly correlated with reduced overall survival $(P=0.0084)$. Ectopic expression of miR-342-3p significantly suppressed proliferation, colony formation, and invasion of NPC cells. In contrast, depletion of miR-342-3p facilitated NPC cell proliferation and invasion. In vivo xenograft studies confirmed that overexpression of miR-342-3p restrained the growth of NPC xenograft tumors. Mechanistically, FOXQ1 served as a functional target of miR-342-3p. There was a significantly negative correlation between miR-342$3 p$ and FOXQ1 expression $(r=-0.487, P=0.004)$ in NPC specimens. Overexpression of FOXQ1 rescued the inhibitory effects of miR-342-3p on NPC cell growth and invasion.

Conclusions: miR-342-3p downregulation predicts poor prognosis in NPC patients and shows suppressive activity against NPC growth and invasion through repression of FOXQ1. Restoration of miR-342-3p may represent a potential therapeutic strategy for NPC.
\end{abstract}

Keywords: Invasion, FOXQ1, Growth, miR-342-3p, Nasopharyngeal carcinoma

\section{Background}

Nasopharyngeal carcinoma (NPC) is a common tumor of the head and neck in Southeast Asia and China [1]. Radiotherapy in combination with chemotherapy is the primary treatment for locally advanced NPC. Due to high recurrence and metastasis rates, the prognosis of advanced NPC is still unsatisfactory, with a 5-year survival rate of approximately $50 \%$ in Chinese population $[2,3]$. However, the exact mechanism for NPC development and progression has not yet been clarified.

Forkhead box (FOX) proteins are a superfamily of transcription factors that are evolutionarily conserved in

\footnotetext{
* Correspondence: zhaoyulinmail@163.com

Department of Rhinology, First Affiliated Hospital of Zhengzhou University, Zhengzhou, China
}

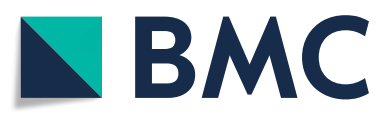

(c) The Author(s). 2019 Open Access This article is distributed under the terms of the Creative Commons Attribution 4.0 International License (http://creativecommons.org/licenses/by/4.0/), which permits unrestricted use, distribution, and

reproduction in any medium, provided you give appropriate credit to the original author(s) and the source, provide a link to the Creative Commons license, and indicate if changes were made. The Creative Commons Public Domain Dedication waiver (http://creativecommons.org/publicdomain/zero/1.0/) applies to the data made available in this article, unless otherwise stated. has been linked to tumorigenesis, metastasis, and drug resistance [5]. For example, FOXO1 phosphorylation overcomes chemoresistance in cancer cells by blocking IQGAP1-MAPK interactions [6]. FOXR2 is required for the growth and spread of prostate cancer cells [7]. Targeted reduction of FOXM1 was found to exert suppressive effects on NPC growth [8]. FOXC2 overexpression induces chemoresistance in NPC cells [9]. FOXQ1 acts as an oncogene in NPC [10]. These studies suggest FOX proteins as promising targets for anticancer treatments.

microRNAs (miRs) are a class of short non-coding RNAs that post-transcriptionally regulate gene expression through base pairing to partially complementary 
sites in the 3'-untranslated region (3'-UTR) of target mRNAs [11]. miRs play a critical role in the growth and progression of cancers [12]. For instance, it was reported that miR-519 inhibits the proliferation of NPC cells by repressing URG4/URGCP [13]. miR-342-3p is frequently dysregulated in human cancers, such as colon cancer [14] and pancreatic cancer [15]. This miR shows growth-suppressive activity in hepatocellular carcinoma [16], lung cancer [17], and gallbladder cancer [18]. miR expression profiling of NPC has revealed the downregulation of miR-342-3p in NPC [19]. However, the biological function of miR-342-3p in NPC is still unknown.

In the present study, we collected 79 paired NPC tissue specimens and adjacent normal tissues and measured the expression of miR-342-3p. The prognostic impact of miR-342-3p was evaluated. To dissect the biological roles of miR-342-3p, gain- and loss-of-function experiments were carried out.

\section{Methods}

\section{Tissue specimens}

We collected 79 paraffin-embedded NPC specimens and their adjacent normal tissues between 2002 and 2008 from the First Affiliated Hospital of Zhengzhou University (Zhengzhou, China). All cases were confirmed by pathological examination. Follow-up data were retrieved from medical records. No patient received anticancer treatment prior to biopsy. There were 51 males and 28 females, with a median age of 56 years (range, 37-85 years). Clinicopathologic characteristics of the patients are shown in Table 1. Written informed consent for research was obtained from each patient. This retrospective study was approved by the Institutional Human Experiment and Ethics Committee of the First Affiliated Hospital of Zhengzhou University.

Table 1 Clinicopathological parameters of NPC patients $(n=79)$

\begin{tabular}{|c|c|c|}
\hline Variable & & No. of cases \\
\hline \multicolumn{3}{|c|}{ Age (years) } \\
\hline$>50$ & & 47 \\
\hline$\leq 50$ & & 32 \\
\hline \multicolumn{3}{|c|}{ Gender } \\
\hline Male & & 51 \\
\hline Female & & 28 \\
\hline \multicolumn{3}{|c|}{ Clinical stage } \\
\hline$|/| \mid$ & & 24 \\
\hline III/IV & & 55 \\
\hline \multicolumn{3}{|c|}{ Lymph node status } \\
\hline Absent & & 19 \\
\hline Present & & 60 \\
\hline
\end{tabular}

\section{Cell culture}

Four human NPC cell lines (C666, SUNE1, HNE1 and CNE2) and NP69 immortalized nasopharyngeal epithelial cells were obtained from the Shanghai Institutes for Biological Sciences, Chinese Academy of Sciences (Shanghai, China). Cells were cultured in RPMI 1640 medium (Thermo Fisher Scientific, Waltham, MA, USA) supplemented with $10 \%$ fetal bovine serum (FBS; Sigma-Aldrich, St. Louis, MO, USA). The identity of the cell lines have been validated by short-tandem repeat analyses. They are free of mycoplasma contamination.

\section{RNA extraction and real-time PCR analysis}

Total RNA was extracted from tissues and cells using TRIzol reagent (Invitrogen, Grand Island, NY, USA). Reverse transcription was performed using the TaqMan Reverse Transcription Kit (Applied Biosystems, Carlsbad, CA, USA) and miR-342-3p specific stem-loop primer. Real-time PCR was conducted using the TaqMan MicroRNA Assay Kit (Applied Biosystems) according to the manufacturer's instructions. U6 was used an endogenous control. The expression of miR-342-3p was normalized against U6.

\section{Stable expression of miR-342-3p in NPC cells}

To generate miR-342-3p stable clones, C666 and CNE2 cells were transfected with a miR-342-3p-expressing plasmid (Cell Biolabs, San Diego, CA, USA) using Lipofectamine 2000 Reagent (Invitrogen). Control cells were transfected with the pEP-miR Null vector (Cell Biolabs). Forty-eight hours post-transfection, cells were selected in the presence of $2 \mu \mathrm{g} / \mathrm{mL}$ puromycin (Sigma-Aldrich) for 10 days.

\section{Transient transfection}

C666 and CNE2 cells were transfected with antimiR-342-3p inhibitor or negative control inhibitor (50 nM; Exiqon, Vedbaek, Denmark) using Lipofectamine 2000 Reagent. In some experiments, a FOXQ1-expressing plasmid or empty vector was transfected to C666 cells with stable expression of miR-342-3p. The FOXQ1-expressing plasmid was generated by inserting a full-length open reading frame of FOXQ1 (lacking the 3'-UTR) into pcDNA3.1(+) expression vector. Twenty-four hours after transfection, cells were tested for gene expression, proliferation, and invasion.

\section{Cell proliferation and colony formation assays}

Cells were seeded onto 96-well plates $\left(4 \times 10^{3}\right.$ cells/well $)$ and cultured for 3 and 5 days. Each well was added with 3-[4,5-dimethyl-2-thiazolyl]-2,5-diphenyl-2H-tetrazolium bromide (MTT) solution $(0.5 \mathrm{mg} / \mathrm{ml}$, Sigma-Aldrich) and incubated for $4 \mathrm{~h}$ at $37^{\circ} \mathrm{C}$. Dimethyl sulfoxide was used to dissolve the resultant crystals. Absorbance was 
recorded at $570 \mathrm{~nm}$. For evaluation of colony formation capacity, cells were plated onto 6-well plates (500 cells/ well) and cultured for 10 days. After staining with 1\% crystal violet (Sigma-Aldrich), the number of colonies was determined using a microscope.

\section{Transwell invasion assay}

We used the methodology previously described by Zhao and colleagues [20]. Cells in serum-free medium containing $10 \mu \mathrm{g} / \mathrm{ml}$ mitomycin C (Sigma-Aldrich) were seeded in the upper chamber of 24-well Transwell plates $\left(2 \times 10^{4}\right.$ cells/well). The inserts $(8 \mu \mathrm{m}$ in pore size $)$ were precoated with Matrigel (BD Biosciences, San Jose, CA, USA). The lower chamber was filled with RPMI 1640 medium containing $10 \%$ FBS. The cells were allowed to invade through the Matrigel-coated inserts for $24 \mathrm{~h}$. Afterwards, invaded cells were stained with $1 \%$ crystal violet and counted under a microscope.

\section{Dual-luciferase reporter assay}

Luciferase reporter assay was performed as previously described [20]. The 3'-UTR of FOXQ1 mRNA was inserted into the pMIR-REPORT Luciferase miRNA Expression Reporter Vector (ThermoFisher Scientific, Waltham, MA, USA). A mutant form with disruption of the miR-342-3p binding site was constructed using the QuikChange site-directed mutagenesis kit (Stratagen, Santa Clara, CA, USA). C666 cells were co-transfected with the reporter constructs together with the miR-342$3 p$-expressing plasmid or empty vector. Luciferase activities were measured using the Dual-Luciferase Reporter Assay System (Promega, Fitchburg, WI, USA) $48 \mathrm{~h}$ after transfection. The relative luciferase activity was determined after normalization against Renilla luciferase activity.

\section{Tumorigenic studies in nude mice}

For tumorigenic studies, C666 and CNE2 cells with stable overexpression of miR-342-3p or empty vector $\left(2 \times 10^{6}\right.$ cells/mouse $)$ were subcutaneously inoculated into the flanks of male BALB/c nude mice (5 weeks of age; Shanghai Laboratory Animals Center of Chinese Academy of Sciences, Shanghai, China). Tumor growth was monitored for 5 weeks. After the last measurement of tumor volume, animals were are euthanized by carbon dioxide inhalation followed by cervical dislocation. The xenograft tumors were resected and weighed. Afterwards, tumor samples were fixed, embedded in paraffin, and subjected to immunostaining for Ki-67 using an anti-Ki-67 monoclonal antibody (Thermo Fisher Scientific; 1:400 dilution). The experiment protocol was approved by the Animal Care and Use Committee of Zhengzhou University.
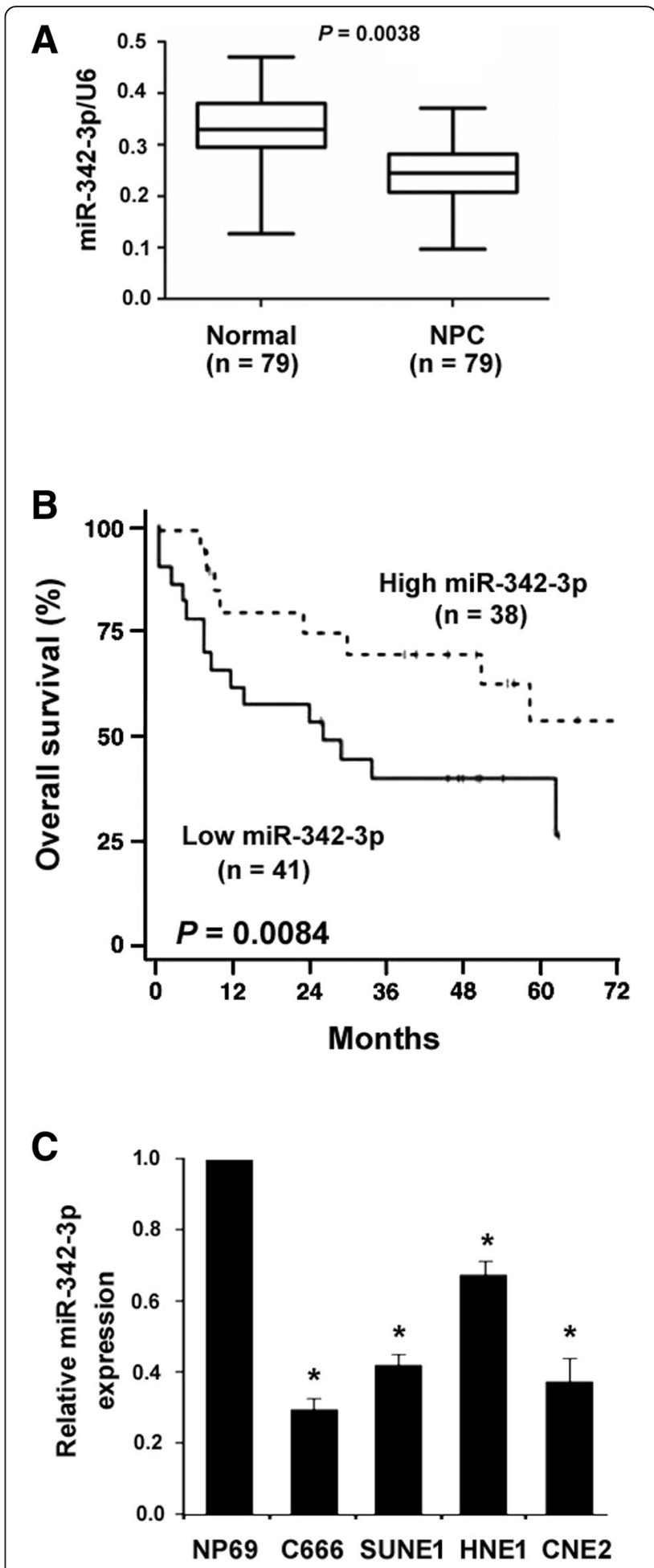

Fig. 1 miR-342-3p is downregulated in NPC and predicts poor prognosis. a Measurement of miR-342-3p levels in 79 paired NPC specimens and adjacent normal nasopharyngeal tissues by real-time PCR analysis. $\mathbf{b}$ Kaplan-Meier curves for overall survival in the NPC patients with high $(n=38)$ vs. low $(n=41)$ miR-342-3p expression. The differences between the survival curves were determined by the log-rank test. c Measurement of miR-342-3p in indicated cell lines. ${ }^{*} P<0.05$ vs. NP69 cells 


\section{Western blot analysis}

Protein samples from NPC tissues and cells were prepared in radioimmunoprecipitation assay buffer (Sigma-Aldrich) supplemented with the Protease and Phosphatase Inhibitor Cocktail (Abcam, Cambridge, UK). Protein samples were separated by SDS-polyacrylamide gel electrophoresis and transferred onto polyvinylidene difluoride membranes. Membranes were probed with rabbit anti-FOXM1 polyclonal antibody (Abcam; 1:500 dilution), anti-FOXQ1 polyclonal antibody (Abcam; 1:500 dilution), or anti$\beta$-actin polyclonal antibody (Thermo Fisher Scientific; 1:5000 dilution), followed by incubation with horseradish peroxidase-conjugated goat anti-rabbit IgG (Sigma-Aldrich). Signals were visualized by enhanced chemiluminescence (Thermo Fisher Scientific). Protein bands were quantified by densitometric analysis using the Quantity One software (Bio-Rad Laboratories, Hercules, CA, USA).

\section{Statistical analysis}

Data are expressed as the mean \pm standard deviation (SD). Differences in the means were determined using the Student's $t$ test or one-way analysis of variance (ANOVA) followed by Tukey's multiple comparison test. The correlation between miR-342-3p and FOXQ1 protein levels in NPC specimens was determined by Pearson's correlation coefficient analysis. Survival curves were plotted using the Kaplan-Meier method and compared using the log-rank test. $P<0.05$ was considered statistically significant.

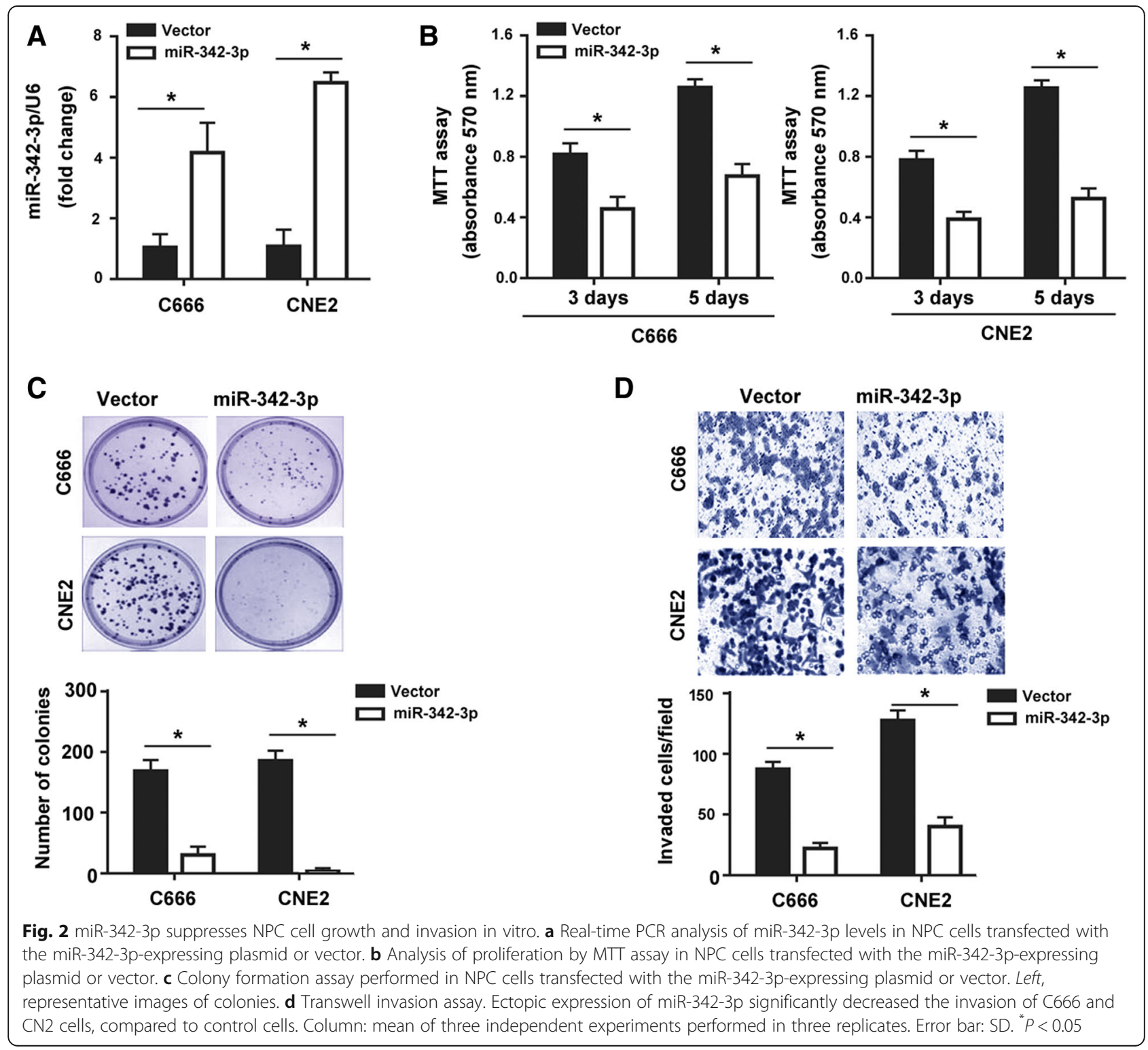




\section{Results}

miR-342-3p is downregulated in NPC and predicts poor prognosis

A total of 79 paired non-malignant and NPC specimens were analyzed for miR-342-3p expression. Compared with corresponding normal nasopharyngeal tissues, NPC tissues displayed a significant downregulation of miR-342-3p $(P=0.0038$; Fig. 1a). According to the median level of miR-324-3p in NPC specimens, the 79 patients were divided into two groups. The prognostic significance of miR-342-3p expression was then evaluated using the Kaplan-Meier method and log-rank tests. It was found that low miR-342-3p levels were significantly correlated with shorter overall survival $(P=0.0084$; Fig. $1 \mathrm{~b})$ of the NPC patients. These results suggest an important role for miR-342-3p in the pathogenesis of NPC.

\section{miR-342-3p suppresses NPC cell growth and invasion in} vitro

Next, we investigated the effect of restoration of miR-342-3p on the aggressive phenotype of NPC cells. Real-time PCR analysis that NPC cell lines including C666 and CNE2 showed significantly lower levels of
miR-342-3p than NP69 non-malignant cells (Fig. 1c). The upregulation of miR-342-3p in both C666 and CNE2 cells stably transfected with the miR-342-3p-expressing plasmid (Fig. 2a). As determined by MTT assay, the number of viable cells was significantly reduced in miR-342-3p-overexpressing cells relative to control cells after culturing for 3 and 5 days $(P<0.05$; Fig. 2b). Colony formation assay further showed that miR-342-3p overexpression reduced the ability of $\mathrm{C} 666$ and CNE2 cells to form colonies $(P<0.05$; Fig. 2c). To determine whether miR-342-3p affects the invasion of NPC cells, Transwell invasion assay was conducted. The results demonstrated that ectopic expression of miR-342-3p significantly inhibited the invasion of $\mathrm{C666}$ and CNE2 cells by 74 and $68 \%$, respectively $(P<0.05$; Fig. 2d).

To further confirm the suppressive roles of miR-342-3p, cell proliferation and invasion were examined after inhibition of miR-342-3p. It was found that transfection with anti-miR-342-3p significantly decreased the expression of miR-342-3p (Fig. 3a), which was accompanied by increased cell proliferation (Fig. 3b) and invasion (Fig. 3c) in both $\mathrm{C666}$ and CNE2 cells.

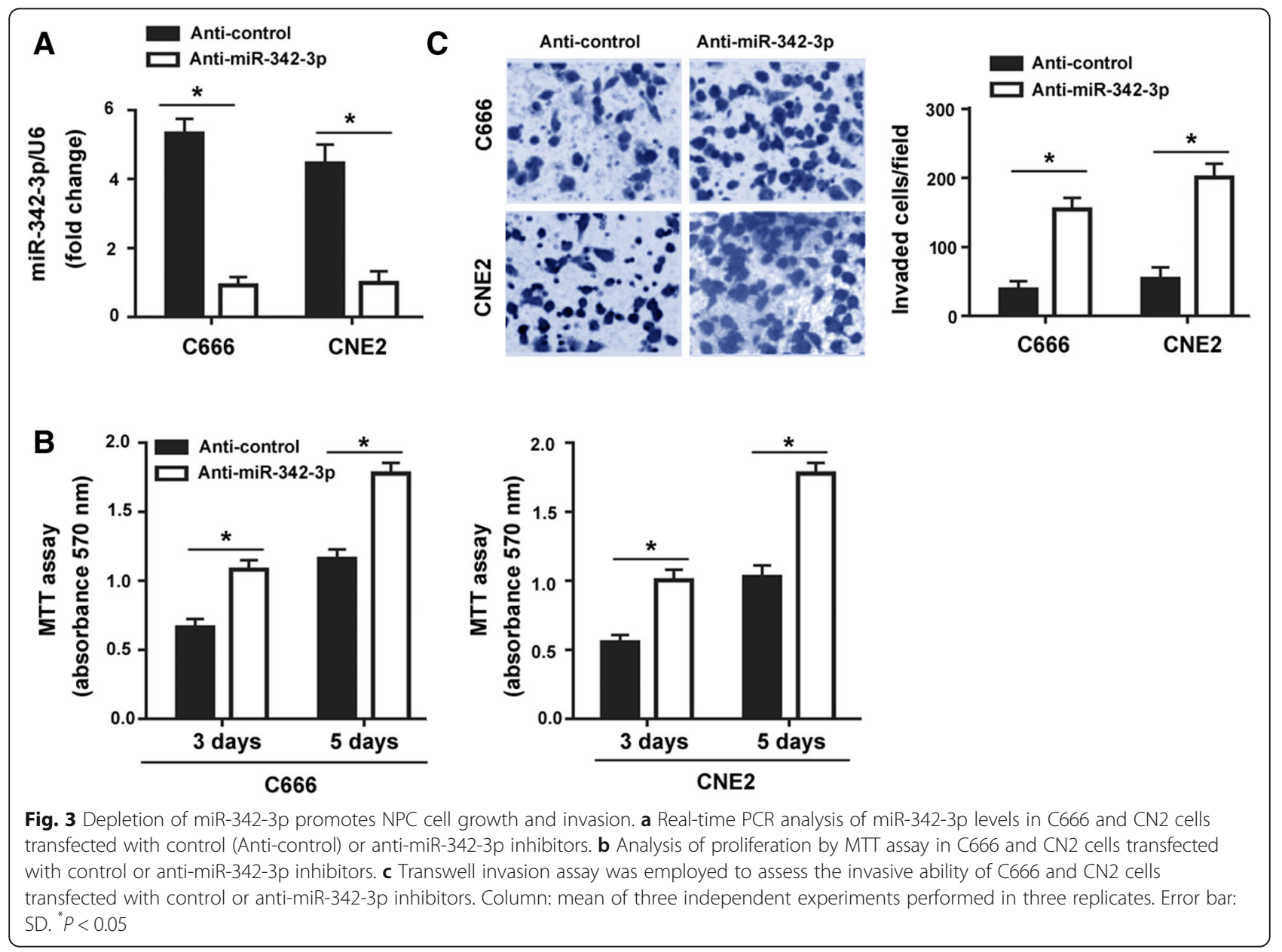




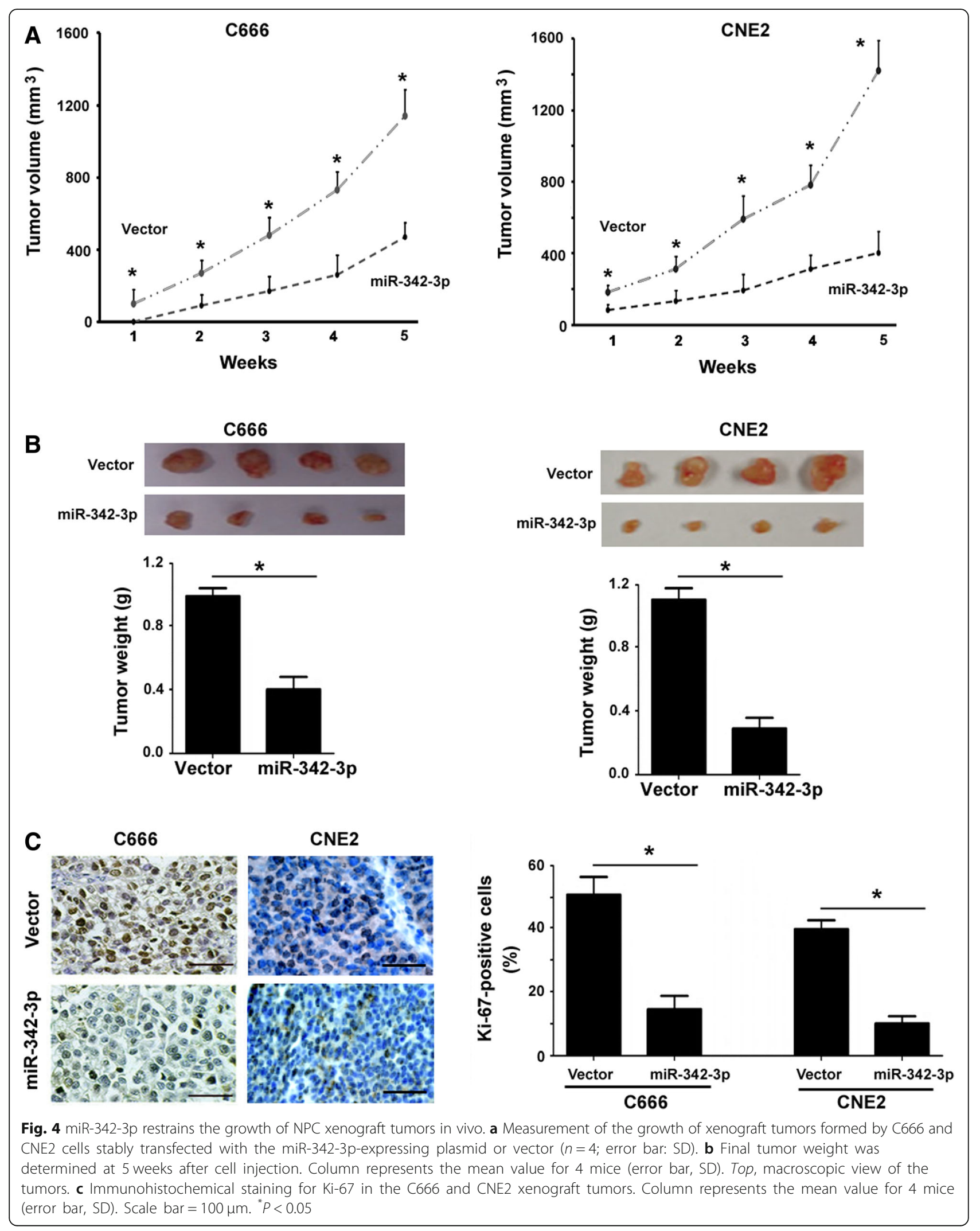




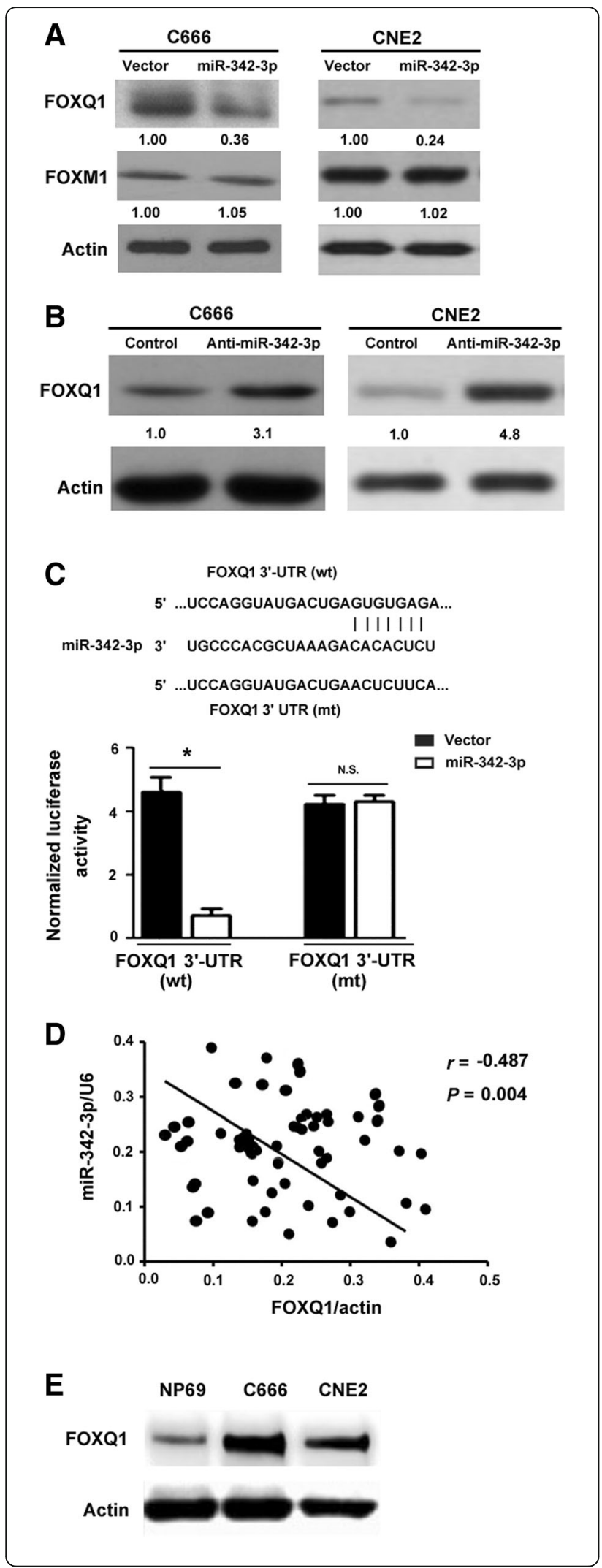

Fig. 5 miR-342-3p targets FOXQ1 in NPC cells. $\mathbf{a}$ and $\mathbf{b}$ Western blot analysis of indicated proteins in C666 and CNE2 cells transfected with indicated constructs. Numbers represent fold change in protein levels. Data represent mean of three independent experiments. c Luciferase reporter assay performed in C666 cells demonstrated that the reporter construct harboring wild-type (wt) FOXQ1 3'-UTR but not the mutant $(\mathrm{mt})$ was suppressed by overexpression of miR-3423p. ${ }^{*} P<0.05$. N.S. indicates no significance. $\mathbf{d}$ Determination of the relationship between miR-342-3p and FOXQ1 protein expression in NPC specimens $(n=79)$ by Pearson's correlation coefficient analysis. e Western blot analysis of FOXQ1 protein levels in indicated cells

miR-342-3p restrains the growth of NPC xenograft tumors in vivo

Next, we assessed the effects of miR-342-3p overexpression on tumor growth in vivo. All the 4 mice injected with empty vector-transfected C666 cells developed a palpable tumor 1 week later, whereas the onset of tumor development was delayed by 1 week in the corresponding miR-342-3p overexpression group (Fig. 4a). Over an observation period of 5 weeks, the tumor growth rate was slower in the miR-342-3p overexpression group than than in the vector group $(P<0.05$; Fig. $4 a)$. When empty vector- or miR-342-3p-transfected CNE2 cells were implanted into nude mice, a minor tumor was palpable at 1 week in both the groups (Fig. 4a). However, there was a marked reduction in the tumor growth rate in the miR-342-3p overexpression group, compared to the corresponding vector group. Final tumor weight was reduced in the miR-342-3p overexpression group (0.39 \pm 0.07 and $0.32 \pm 0.06 \mathrm{~g}$ in C666 and CNE2 tumors, respectively) than in the control group $(0.88 \pm 0.04$ and $1.12 \pm 0.08 \mathrm{~g}$ in $\mathrm{C} 666$ and CNE2 tumors, respectively; Fig. 4b). Immunohistochemical staining confirmed that overexpression of miR-342-3p led to a significant decline in the percentage of Ki-67-positive cells in the xenograft tumors (Fig. 4c). These data indicated that miR-342-3p shows growth-suppressive activity in NPC cells in vivo.

miR-342-3p targets FOXQ1 in NPC cells

It has been documented that both FOXM1 and FOXQ1 serve as two targets of miR-342-3p in colorectal cancer [21]. Given the importance of FOX proteins in NPC development, we tested whether miR-342-3p exerts its suppressive activity through targeting of FOXM1 and FOXQ1. Western blot analysis showed that overexpression of miR-342-3p markedly downregulated the expression of FOXQ1, but had little effect on FOXM1 expression in C666 cells (Fig. 5a). In contrast, delivery of anti-miR-342-3p promoted the expression of FOXQ1 (Fig. 5b) in C666 cells. Similar findings were observed in CNE2 cells (Fig. 5a and b). Luciferase reporter assay showed that overexpression of miR-342-3 p significantly suppressed the wild-type FOXQ1 3'-UTR reporter construct, but not the mutant form (Fig. $5 \mathrm{c}$ ). We measured 


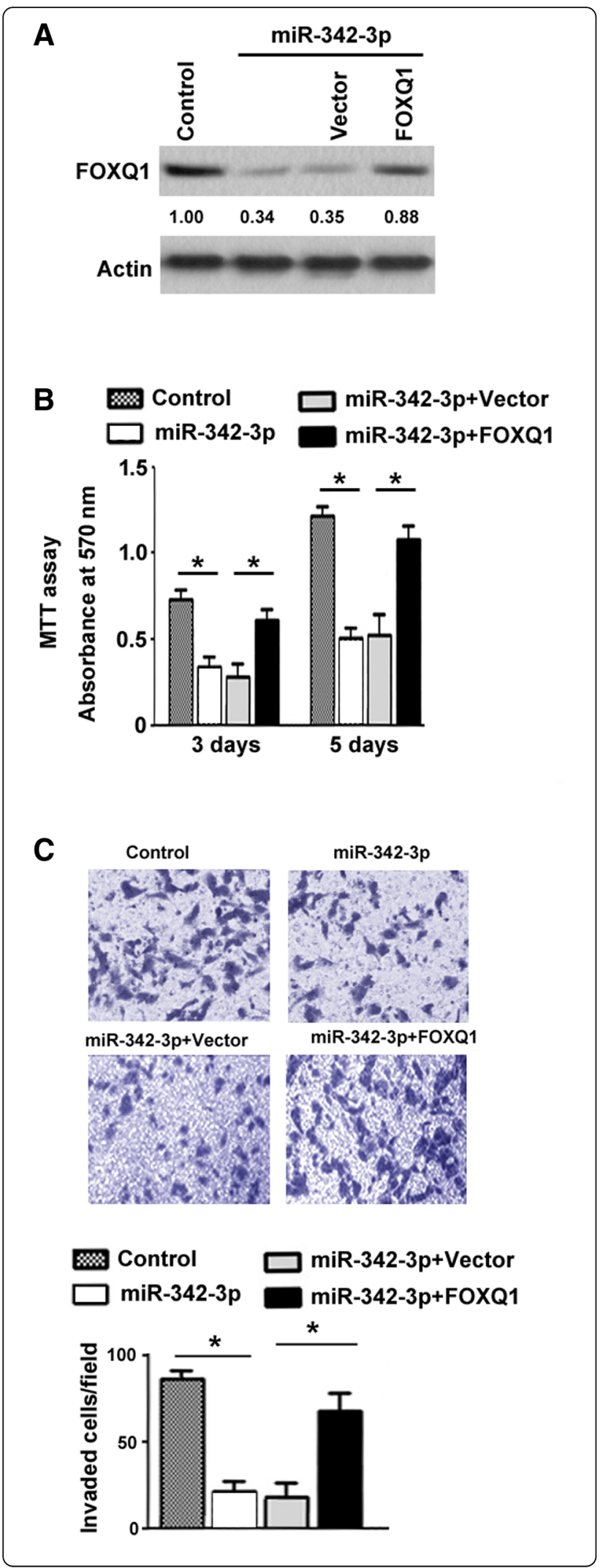

Fig. 6 FOXQ1 rescues NPC cells from miR-342-3p-induced growth and invasion suppression. a Western blot analysis of FOXQ1 protein levels in C666 cells co-transfected with miR-342-3p- and FOXQ1expressing plasmid or vector. Numbers represent fold change in protein levels. b Analysis of proliferation by MTT assay in C666 cells transfected with indicated constructs. c Transwell invasion assay was employed to assess the invasive ability of C666 cells transfected with indicated constructs. Top, representative images of invaded cells. Column: mean of three independent experiments performed in three replicates. Error bar: SD. ${ }^{*} P<0.05$

the protein levels of FOXQ1 in NPC specimens by Western blotting. Analysis of the relationship between miR-342-3p and FOXQ1 expression in NPC specimens revealed that there was a significantly negative correlation between miR-342-3p and FOXQ1 expression $(r=-0.487, P=0.004$; Fig. $5 \mathrm{~d})$. However, no significant correlation was detected between the expression of miR-342-3p and FOXM1 in NPC tissues $(P>0.05)$. In addition, we found an upregulation of FOXQ1 in both C666 and CNE2 cells compared to NP69 cells (Fig. 5e). These data suggest that FOXQ1 is a direct target of miR-342-3p in NPC.

FOXQ1 rescues NPC cells from miR-342-3p-induced growth and invasion suppression

Having identified the downregulation of FOXQ1 by miR-342-3p, we next addressed whether enforced expression of FOXQ1 can rescue the suppressive effect of miR-342-3p on NPC cells. To this end, we overexpressed a miR-resistant variant of FOXQ1 in C666 cells together with ectopic expression of miR-342-3p (Fig. 6a). Overexpression of FOXQ1 significantly restored the proliferation (Fig. 6b) and invasion (Fig. 6c) capacity of miR-342-3p-overexpressing C666 cells. These observations suggest that FOXQ1 mediates the activity of miR-342-3p in the regulation of NPC growth and invasion.

\section{Discussion}

In this study, we showed that miR-342-3p was significantly underexpressed in NPC tissues compared to adjacent normal nasopharyngeal tissues. In line with our findings, the dysregulation of miR-342-3p is also described in colon cancer [14], pancreatic cancer [15], and hepatocellular carcinoma [22]. We further analyzed the relationship between miR-342-3p expression and survival of NPC patients. It was found that downregulation of miR-342-3p had a poor prognostic impact on the survival of patients with NPC. Similarly, low miR-342-3p expression is significant associated with reduced overall survival in patients with hepatocellular carcinoma [22]. These observations favor the idea that miR-342-3p may govern the progression of NPC. 
To clarify the biological relevance of miR-342-3p downregulation in NPC, we performed gain- and lossof-function experiments. The results demonstrated that ectopic expression of miR-342-3p significantly suppressed proliferation, colony formation, and invasion of NPC cells. In contrast, depletion of miR-342-3p led to a significant enhancement of NPC cell proliferation and invasion. In vivo tumorigenic studies confirmed that miR-342-3p overexpression hampered the growth of NPC xenograft tumors. These data indicate that miR342-3p acts as a tumor suppressor in NPC. In support of our point, several previous studies have documented that miR-342-3p plays a tumor-suppressive role in multiple malignancies including hepatocellular carcinoma [16], colorectal cancer [21], osteosarcoma [23], and small cell lung cancer [24]. However, a recent study has provided evidence that miR-342-3p has no significant impact on the progression of pancreatic acinar carcinoma in a mouse model [25]. Therefore, the functional consequence of miR-342-3p depends on cellular contexts.

It is well accepted that a single miR has the ability to modulate a large number of target genes [11]. Different targets have been identified to mediate distinct biological activities of miR-342-3p [23, 26, 27]. For instance, miR-342-3p regulates lumen formation in mammary gland morphogenesis through repression of ID4 and DNMT1 [26]. This miR shows the ability to induce adipogenesis of mesenchymal stem cells by suppressing CtBP2 [27]. Both FOXM1 and FOXQ1 mediate the tumor-suppressive activity of miR-342-3p in colorectal cancer cells [21]. Similarly, we found that miR-342-3p overexpression caused a significant downregulation of FOXQ1 in both C666 and CNE2 cells. However, the level of FOXM1 remained unchanged in response to miR-342-3p overexpression. The selective repression of FOXQ1 implies its critical role in mediating the activity of miR-342-3p in NPC. Clinical evidence indicates a negative correlation between miR-342-3p and FOXQ1 expression in NPC specimens. Rescue experiments using a miR-resistant variant of FOXQ1 demonstrated that enforced expression of FOXQ1 significantly reversed the suppressive effect of miR-342-3p on NPC cell proliferation and invasion. FOXQ1 functions as an oncogene in colorectal carcinoma [28], gastric cancer [29], and NPC [10]. It has been documented that FOXQ1 can facilitate the metastasis of gastric cancer cells through upregulation of Snail [29]. FOXQ1 is upregulated in NPC and can be regulated by a number of miRs including miR-124 [10] and miR-506 [30]. Our data expand the list of miR regulators of FOXQ1 to include miR-342-3p and confirm the upregulation of FOXQ1 as a consequence of dysregulation of miRs in NPC.

However, it should be mentioned that apart from FOXQ1, other target genes may also be involved in the activity of miR-342-3p in NPC. Genome-wide identification of target genes would be necessary to uncover the mechanism by which miR-342-3p governs the aggressive phenotype of NPC cells. The mechanism underlying the downregulation of miR-342-3p in NPC is still elusive. A previous study has indicated that long non-coding RNAs such as FTX [31] show the capacity to repress the expression of miR-342-3p. The reduced expression of miR-342-3p can also be explained as a result of abberrant methylation of the promoter region of its host gene, EVL [32]. Ongoing studies are conducted to address this issue. Another limitation of this study was the enrollment of patients from a single center.

\section{Conclusions}

In summary, our data demonstrate that miR-342-3p has a poor prognostic impact on the survival of NPC patients. Restoration of miR-342-3p suppresses NPC cell growth and invasion via targeting of FOXQ1 and may have therapeutic benefits in the treatment of this malignancy.

\section{Abbreviations \\ 3'-UTR: 3'-untranslated region.; FOX: forkhead box; miRs: microRNAs; NPC: nasopharyngeal carcinoma \\ Acknowledgements \\ None. \\ Funding \\ No funding was obtained for this study. \\ Availability of data and materials \\ The datasets used and/or analysed during the current study are available from the corresponding author on reasonable request.}

Authors' contributions

ZC and $Y Z$ made substantial contributions to conception design, acquisition of data, analysis and interpretation of data, and write/edit manuscript. All authors have read and approved the final manuscript.

Ethics approval and consent to participate

Written informed consent for research was obtained from each patient. This retrospective study was approved by the Institutional Human Experiment and Ethics Committee of the First Affiliated Hospital of Zhengzhou University.

\section{Consent for publication}

Not applicable.

\section{Competing interests}

The authors declare that they have no competing interest.

\section{Publisher's Note}

Springer Nature remains neutral with regard to jurisdictional claims in published maps and institutional affiliations.

Received: 2 April 2018 Accepted: 13 December 2018

Published online: 24 January 2019

\section{References}

1. Dai W, Zheng H, Cheung AK, Lung ML. Genetic and epigenetic landscape of nasopharyngeal carcinoma. Chin Clin Oncol. 2016;5:16. 
2. Wang SY, Lou JL, Chen J, Zhang SZ, Guo L. Salvage surgery for neck residue or recurrence of nasopharyngeal carcinoma after primary radiotherapy: options of surgical methods and regions. World J Surg Oncol. 2016;14:89.

3. Lv JW, Huang XD, Chen YP, Zhou GQ, Tang LL, Mao YP, et al. A National Study of survival trends and conditional survival in nasopharyngeal carcinoma: analysis of the National Population-Based Surveillance Epidemiology and end results registry. Cancer Res Treat. 2018;50:324-34.

4. Pan X, Zhang Y, Kim HG, Liangpunsakul S, Dong XC. FOXO transcription factors protect against the diet-induced fatty liver disease. Sci Rep. 2017;7:44597.

5. Hannenhalli S, Kaestner $\mathrm{KH}$. The evolution of fox genes and their role in development and disease. Nat Rev Genet. 2009;10:233-40.

6. Pan CW, Jin X, Zhao Y, Pan Y, Yang J, Karnes RJ, et al. AKT-phosphorylated FOXO1 suppresses ERK activation and chemoresistance by disrupting IQGAP1-MAPK interaction. EMBO J. 2017;36:995-1010.

7. Xu W, Chang J, Liu G, Du X, Li X. Knockdown of FOXR2 suppresses the tumorigenesis, growth and metastasis of prostate cancer. Biomed Pharmacother. 2017;87:471-5.

8. Chen H, Yang C, Yu L, Xie L, Hu J, Zeng L, et al. Adenovirus-mediated RNA interference targeting FOXM1 transcription factor suppresses cell proliferation and tumor growth of nasopharyngeal carcinoma. J Gene Med. 2012:14:231-40

9. Zhou Z, Zhang L, Xie B, Wang X, Yang X, Ding N, et al. FOXC2 promotes chemoresistance in nasopharyngeal carcinomas via induction of epithelial mesenchymal transition. Cancer Lett. 2015;363:137-45.

10. Peng XH, Huang HR, Lu J, Liu X, Zhao FP, Zhang B, et al. MiR-124 suppresses tumor growth and metastasis by targeting Foxq1 in nasopharyngeal carcinoma. Mol Cancer. 2014;13:186.

11. Wang J, Ye H, Zhang D, Cheng K, Hu Y, et al. Cancer-derived circulating MicroRNAs promote tumor angiogenesis by entering dendritic cells to degrade highly complementary MicroRNAs. Theranostics. 2017;7:1407-21.

12. Jia $L, X i$, Wang $H$, Zhang Z, Liu H, Cheng Y, et al. miR-142-5p regulates tumor cell PD-L1 expression and enhances anti-tumor immunity. Biochem Biophys Res Commun. 2017:488:425-31.

13. Yu G, Zhang T, Jing Y, Bao Q, Tang Q, Zhang Y. miR-519 suppresses nasopharyngeal carcinoma cell proliferation by targeting oncogene URG4/ URGCP. Life Sci. 2017;175:47-51.

14. Tao K, Yang J, Guo Z, Hu Y, Sheng H, Gao H, et al. Prognostic value of miR221-3p, miR-342-3p and miR-491-5p expression in colon cancer. Am J Trans Res. 2014;6:391-401.

15. Lee KH, Lee JK, Choi DW, Do IG, Sohn I, Jang KT, et al. Postoperative prognosis prediction of pancreatic cancer with seven microRNAs. Pancreas. 2015;44:764-8

16. Zhao L, Zhang Y. miR-342-3p affects hepatocellular carcinoma cell proliferation via regulating NF-kB pathway. Biochem Biophys Res Commun. 2015;457:370-7.

17. Xie $X$, Liu H, Wang M, Ding F, Xiao H, Hu F, et al. miR-342-3p targets RAP2B to suppress proliferation and invasion of non-small cell lung cancer cells. Tumour Biol. 2015;36:5031-8.

18. Wang $\mathrm{SH}, \mathrm{Ma}$ F, Tang $\mathrm{ZH}$, Wu XC, Cai Q, Zhang MD, et al. Long noncoding RNA H19 regulates FOXM1 expression by competitively binding endogenous miR-342-3p in gallbladder cancer. J Exp Clin Cancer Res. 2016;35:160.

19. Li T, Chen JX, Fu XP, Yang S, Zhang Z, Chen KH, et al. microRNA expression profiling of nasopharyngeal carcinoma. Oncol Rep. 2011;25:1353-63.

20. Zhao L, Liu L, Dong Z, Xiong J. miR-149 suppresses human non-small cell lung cancer growth and metastasis by inhibiting the FOXM1/cyclin D1/ MMP2 axis. Oncol Rep. 2017:38:3522-30.

21. Weng W, Okugawa Y, Toden S, Toiyama Y, Kusunoki M, Goel A. FOXM1 and FOXQ1 are promising prognostic biomarkers and novel targets of tumor-suppressive miR-342 in human colorectal Cancer. Clin Cancer Res. 2016;22:4947-57.

22. Gao Y, Zhang SG, Wang ZH, Liao JC. Down-regulation of miR-342-3p in hepatocellular carcinoma tissues and its prognostic significance. Eur Rev Med Pharmacol Sci. 2017;21:2098-102.

23. Zhang S, Liu L, Lv Z, Li Q, Gong W, Wu H. MicroRNA-342-3p inhibits the proliferation, migration, and invasion of osteosarcoma cells by targeting astrocyte-elevated Gene-1 (AEG-1). Oncol Res. 2017. https://doi.org/10.3727/ $096504017 X 14886485417426$ [Epub ahead of print].

24. Xu H, Cai T, Carmona GN, Abuhatzira L, Notkins AL. Small cell lung cancer growth is inhibited by miR-342 through its effect of the target gene IA-2.J Transl Med. 2016;14:278.
25. Dooley J, Lagou V, Pasciuto E, Linterman MA, Prosser HM, Himmelreich U, et al. No functional role for microRNA-342 in a mouse model of pancreatic acinar carcinoma. Front Oncol. 2017;7:101. https://doi.org/10.3389/fonc.2017. 00101 eCollection 2017.

26. Weng C, Nguyen T, Shively JE. miRNA-342 Regulates CEACAM1-induced Lumen Formation in a Three-dimensional Model of Mammary Gland Morphogenesis. J Biol Chem. 2016;291:16777-86.

27. Wang L, Xu L, Xu M, Liu G, Xing J, Sun C, et al. Obesity-associated MiR-342$3 p$ promotes Adipogenesis of mesenchymal stem cells by suppressing CtBP2 and releasing C/EBPa from CtBP2 binding. Cell Physiol Biochem. 2015:35:2285-98.

28. Liu JY, WU XY, Wu GN, Liu FK, Yao XQ. FOXQ1 promotes cancer metastasis by PI3K/AKT signaling regulation in colorectal carcinoma. Am J Transl Res. 2017;9:2207-18.

29. Zhang J, Liu Y, Zhang J, Cui X, Li G, Wang J, et al. FOXQ1 promotes gastric cancer metastasis through upregulation of snail. Oncol Rep. 2016;35:3607-13.

30. Zhang Z, Ma J, Luan G, Kang L, Su Y, He Y, et al. MiR-506 suppresses tumor proliferation and invasion by targeting FOXQ1 in nasopharyngeal carcinoma. PLoS One. 2015;10:e0122851.

31. Zhang W, Bi Y, Li J, Peng F, Li H, Li C, et al. Long noncoding RNA FTX is upregulated in gliomas and promotes proliferation and invasion of glioma cells by negatively regulating miR-342-3p. Lab Investig. 2017;97:447-57.

32. Li Z, Wong KY, Chan GC, Chng WJ, Chim CS. Epigenetic silencing of EVL/ miR-342 in multiple myeloma. Transl Res. 2018;192:46-53.
Ready to submit your research? Choose BMC and benefit from:

- fast, convenient online submission

- thorough peer review by experienced researchers in your field

- rapid publication on acceptance

- support for research data, including large and complex data types

- gold Open Access which fosters wider collaboration and increased citations

- maximum visibility for your research: over $100 \mathrm{M}$ website views per year

At BMC, research is always in progress.

Learn more biomedcentral.com/submissions 